\title{
Modeling neurological diseases with induced pluripotent cells reprogrammed from immortalized lymphoblastoid cell lines
}

Koki Fujimori ${ }^{1 \dagger}$, Toshiki Tezuka ${ }^{1 \dagger}$, Hiroyuki Ishiura ${ }^{2}$, Jun Mitsui ${ }^{2}$, Koichiro Doi ${ }^{3}$, Jun Yoshimura ${ }^{3}$, Hirobumi Tada ${ }^{4,5}$, Takuya Matsumoto ${ }^{1,6}$, Miho Isoda $^{1}$, Ryota Hashimoto ${ }^{7,8}$, Nubutaka Hattori ${ }^{9}$, Takuya Takahashi ${ }^{4}$, Shinichi Morishita ${ }^{3,10,}$ Shoji Tsuji ${ }^{2,10}$, Wado Akamatsu ${ }^{1,11^{*}}$ and Hideyuki Okano ${ }^{1 *}$

\begin{abstract}
Patient-specific induced pluripotent stem cells (iPSCs) facilitate understanding of the etiology of diseases, discovery of new drugs and development of novel therapeutic interventions. A frequently used starting source of cells for generating iPSCs has been dermal fibroblasts (DFs) isolated from skin biopsies. However, there are also numerous repositories containing lymphoblastoid B-cell lines (LCLs) generated from a variety of patients. To date, this rich bioresource of LCLs has been underused for generating iPSCs, and its use would greatly expand the range of targeted diseases that could be studied by using patient-specific iPSCs. However, it remains unclear whether patient's LCL-derived iPSCs (LiPSCs) can function as a disease model. Therefore, we generated Parkinson's disease patient-specific LiPSCs and evaluated their utility as tools for modeling neurological diseases. We established iPSCs from two LCL clones, which were derived from a healthy donor and a patient carrying PARK2 mutations, by using existing non-integrating episomal protocols. Whole genome sequencing (WGS) and comparative genomic hybridization (CGH) analyses showed that the appearance of somatic variations in the genomes of the iPSCs did not vary substantially according to the original cell types (LCLS, T-cells and fibroblasts). Furthermore, LiPSCs could be differentiated into functional neurons by using the direct neurosphere conversion method (dNS method), and they showed several Parkinson's disease phenotypes that were similar to those of DF-iPSCs. These data indicate that the global LCL repositories can be used as a resource for generating iPSCs and disease models. Thus, LCLs are the powerful tools for generating iPSCs and modeling neurological diseases.
\end{abstract}

Keywords: Lymphoblastoid B-cell line, Disease modeling, Neurological disorder, Induced pluripotent stem cells, Genomic mutation in reprogramming process

\section{Introduction}

Modeling with induced pluripotent cells (iPSCs) is highly useful in research of neural diseases [1-4] because it is difficult to obtain patient-derived cells from the central nervous system that recapitulates the disease pathology. Dermal fibroblasts (DFs) have widely been used as a source of patient-specific iPSCs because they

\footnotetext{
* Correspondence: awado@juntendo.ac.jp; hidokano@a2.keio.jp

${ }^{\dagger}$ Equal contributors

'Department of Physiology, Keio University, School of Medicine, Shinjuku-ku,

Tokyo 160-8582, Japan

Full list of author information is available at the end of the article
}

were used to derive the first human iPSCs [5]. However, the isolation of DF cell lines requires invasive skin biopsies from patients. Recently, we have reported that T-cell derived iPSCs (TiPSCs) and fibroblast-derived iPSCs can be used to model neural diseases by using robust neural induction protocols [6]. TiPSCs can be established from a small amount of peripheral blood, such that patients can provide samples through a minimally invasive procedure [7]. Although peripheral blood cells, including T-cells, appear to be ideal sources for generating iPSCs, cloned and frozen T-cells are unstable, owing to their variable reprogramming efficiency depending on the 
donors and conditions of each sample (e.g., culture medium or number of passages) [8-10]. In particular, efficient wide-scale parallel reprogramming of samples requires a stable source of cells to achieve highthroughput processing and to model polygenic or sporadic diseases. Lymphoblastoid B-cell lines (LCLs) are stable peripheral B-cell lines that are transformed by infection with Epstein-Barr virus (EBV). LCLs are easy to maintain, and various types of well-characterized LCL clones established from patients are already available in worldwide repositories and are usually linked to patient clinical history, long-term genotype and phenotype data, and molecular/functional studies of various diseases $[11,12]$. LCL banks have been of great importance in providing reference material for rare genetic diseases, as well as in managing large amounts of DNA for the genetic analysis of complex conditions in populationand family-based disease collections [13, 14]. A number of major facilities currently establish and manage extensive collections of cell lines (https://catalog.coriell.org/; http://ja.brc.riken.jp/; https://www.eagle-i.net/; www.ecacc.org.uk; www.alspac.bris.ac.uk; www.lgcpromochem-atcc.com; www.rutgers.edu) for the international research community.

To date, five groups have reported the successful reprogramming of LCLs from healthy donors and patients into iPSCs [15-19]. However, it is unclear whether LCL-derived iPSCs (LiPSCs) can be used for disease-specific analyses. Although immortalization of B-cells by EBV infection [20] may be a problematic procedure in terms of the cellular characteristics of LiPSCs, little is known about the effects of EBV infection on the properties of LiPSCs or even LCLs. Although EBV transformation commonly maintains the genome composition of the original cells [21], long-term LCL culture may cause several aberrations, including genomic aberrations such as chromosomal aneuploidy, down-regulation of $\mathrm{p} 16 / \mathrm{Rb}$, mutation of the p53 gene, modulation of apoptosis and sensitivity to various chemical agents [22]. Therefore, detailed analyses of the genomic structure and cellular properties of LCLs and LiPSCs are necessary because unexpected genomic mutations would confound the diseasespecific phenotypes in disease models generated with iPSCs. Additionally, some genomic aberrations and/or epigenetic memories derived from the source cells can affect the differentiation ability of iPSCs [23-25].

Although neurological disease models generated with LiPSCs would accelerate the progress of patient-specific iPSC studies, these concerns regarding the effects of EBV must be elucidated. Here, we established LiPSCs from both healthy donor and patient with the mutation of PARK2 (parkin) known as one of the causative genes for familial Parkinson's disease,) to evaluate the characteristics of LiPSCs, including whole genomic sequencing and to confirm the utility of LiPSCs as tools for modeling neurological diseases.

\section{Results}

Establishment and characterization of iPSCs derived from LCLs

Previous reports have shown that reprogramming LCLs to iPSCs by using existing non-integrating episomal protocols provided no identifiable iPSC clones, even after 35-40 days [17, 18]. Recently, R. Barrett and colleagues have developed a new episomal (OriP/EBNA1) plasmid reprogramming method for LCLs, which reduces the time required to generate LiPSCs and enhances the efficiency of reprograming LCLs into iPSCs [15]. We modified this method for on-feeder cultures and applied it to LCLs from two donors: a healthy person, "KA" and a patient with autosomal recessive juvenile Parkinson's disease (PARK2), "PB" (Fig. 1a). LiPSCs were generated with a similar efficiency to that of a previously described method [15], and the reprogramming efficiencies of LKA and LPB were 0.002 and $0.0015 \%$, respectively. After optimizing the protocol for generating iPSCs from LCLs, we established several LiPSC clones, including three healthy donor-derived LiPSC lines, LKA10, LKA29, and LKA36 and four PARK2 patient-derived LiPSC lines, LPB1, LPB3, LPB7 and LPB8, which had a normal karyotype (Additional file 1: Figure S1A). In addition, we used four DF-iPSC lines derived from the same healthy donor, eKA3, eKA4, KA11 and KA23, as the control reference lines [6] in this study.

Representative morphologies of LiPSC colonies are shown in Fig. 1b. All LiPSC lines exhibited typical PSC characteristics, including tightly packed colonies, a high cell nuclear-cytoplasmic ratio, and the production of the surface and nuclear pluripotency proteins, TRA1-60 and OCT4 and were indistinguishable from the DF-iPSC lines (Fig. 1b). LiPSCs also expressed endogenous pluripotency genes at a similar level to DF-iPSCs (Figs. 1c-e). These data indicated that LiPSCs and DF-iPSCs were indistinguishable in terms of morphology and the expression of pluripotent markers at both the protein and mRNA levels (Fig. 1b-e). In addition, we confirmed whether LiPSCs have differentiation potentials into three-germ layers by in vitro differentiation analysis via EB (Additional file 1: Figure S1B and C). Thus, all LiPSC clones had a pluripotency. Because EBNA-1 has been reported to be required for the establishment of persistent EBV infection and survival of host B-cells [26], we next examined the expression of EBNA1 and additional EBVrelated genes (EBNA-2, BZLF-1, LMP-1 and OriP) in LiPSCs. A PCR analysis of genomic DNA showed that all the EBV-related latency elements were eventually eliminated from the established LiPSCs, suggesting the 




B
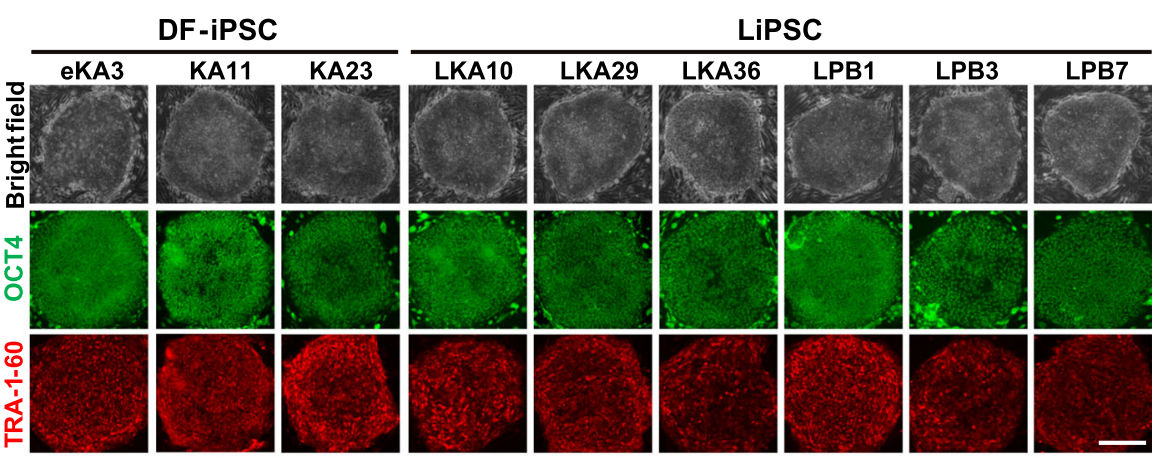

C

NANOG

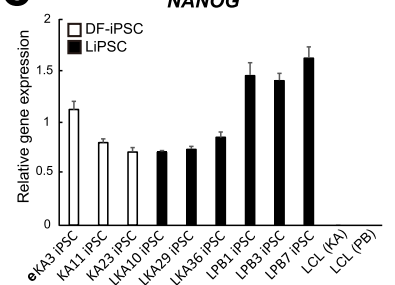

D
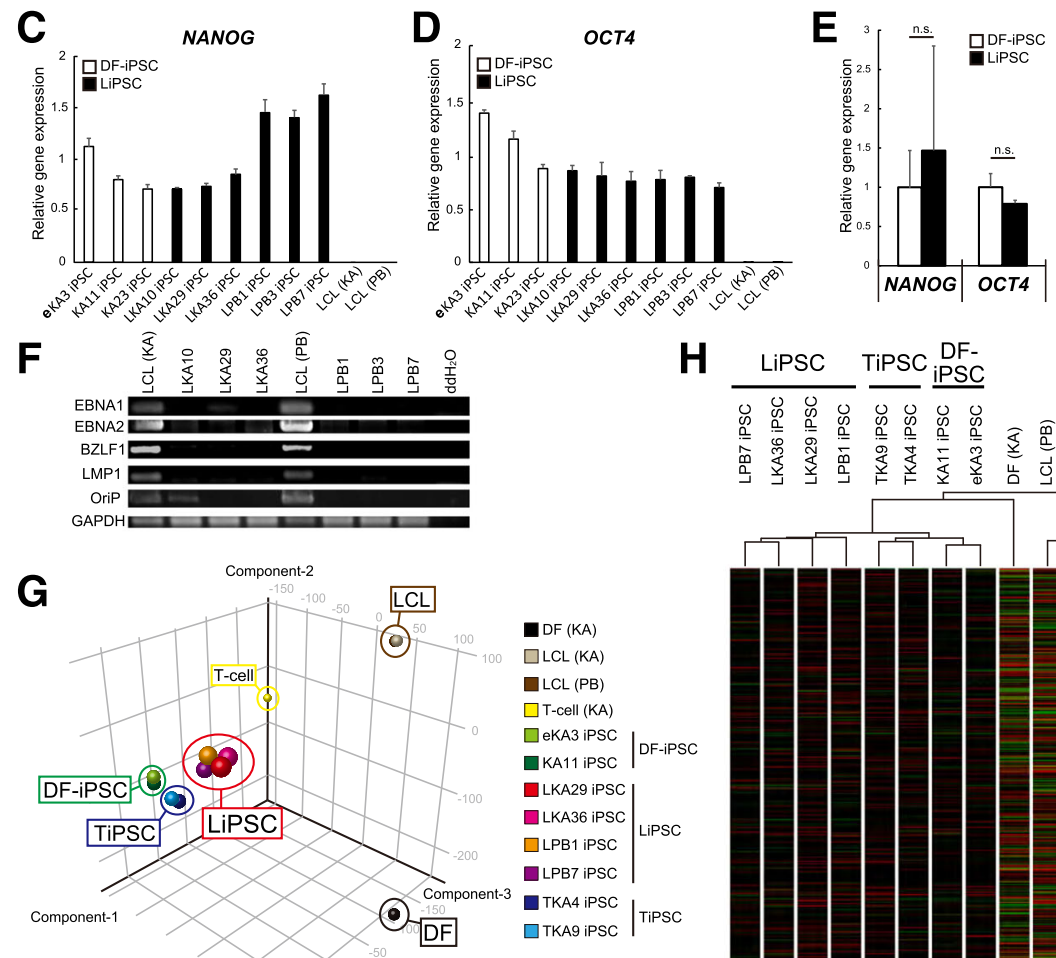

H
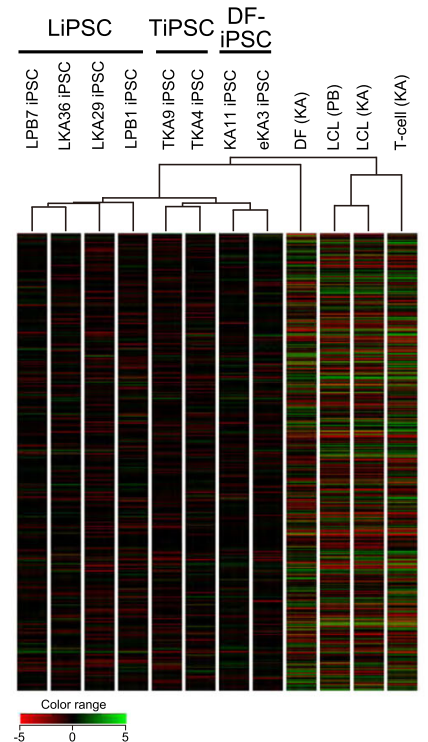

Fig. 1 Establishment and characterization of LCL-derived iPSCs. a Representative image of an LCL culture. Scale bar $=100 \mu \mathrm{m}$. $\mathbf{b}$ iPSCs derived from healthy control LCLs (LKA10, LKA29 and LKA36), isogenic dermal fibroblasts (DFs) (eKA3, KA11, and KA23) and LCLs from a PARK2 patient (LPB1, LPB3 and LPB7) were immunopositive for the pluripotent markers OCT4 (green) and TRA-1-60 (red). Scale bar = 200 $\mu m$. c-e The expression levels of pluripotent markers NANOG and OCT4 in LiPSCs (LKA10, LKA29 LKA36, LPB1, LPB3 and LPB7), DF-iPSCs (eKA3, KA11 and KA23) and LCLs $(L C L(K A)$ and $L C L(P B))$ were assessed by quantitative reverse-transcription PCR (qPCR). The values from the previously established DF-iPSCs (201B7, a previously established human iPSC clone [5]) were set to 1.0 ( $n=3$ independent experiments; means \pm SEM; $n$.S., not significant; Student's $t$-test). f The expression levels of EBV-related genes (EBNA-1, EBNA-2, BZLF-1, LMP-1 and OriP) were analyzed by a PCR analysis of the genomic DNA obtained from parental LCLs and LCL-derived iPSCS. GAPDH was used a loading control. $\mathbf{g}$ Comparison of the global gene expression profiles of DF-iPSCS (eKA3 and KA11), LiPSCs (LKA29, LKA36, LPB1 and LPB7), TiPSCs (TKA4 and TKA9) [6], and the original cells (DF(KA), LCL(KA), LCL(PB) and T-cell(KA)). Principal component analysis of the gene expression data. Black: DF, Brown: LCLs, Yellow: T-cell, Green: DF-iPSCs, Red: LiPSCs, Blue: TiPSCs. h Hierarchical clustering analysis of the global gene expression profiles. The data discussed in this publication have been deposited in the NCBI Gene Expression Omnibus (GEO, http://www.ncbi.nlm.nih.gov/geo/) database and are accessible through GEO Series accession numbers GSE76832 [6] and GSE82159 
loss of EBV-associated elements as a result of the reprogramming process (Fig. 1f).

The global gene expression profiles in iPSCs at passages below 20 were evaluated with a microarray analysis to explore the detailed differences between LiPSCs, TiPSCs and DF-iPSCs resulting from the origin of the iPSCs. With the exception of the genes that were expressed at low levels in all samples, the data were normalized and subjected to principal component analysis (PCA) (Fig. 1g) and hierarchical clustering (Fig. 1h). Although the LiPSCs, TiPSCs and DF-iPSCs were relatively close to each other in the PCA analysis (Fig. 1g), hierarchical clustering placed the LiPSCs, TiPSCs and DFiPSCs into different groups (Fig. 1h). These data also suggested that the original cell type influences the properties of hiPSCs.

Immortalization by EBV does not affect the number of $d e$ novo mutations and structural variations in LiPSCs

We performed array-based comparative genomic hybridization $(\mathrm{aCGH})$ and whole genome sequence (WGS) analyses to examine the somatic structural variations (SVs) and single nucleotide variations (SNVs) in LiPSCs (Fig. 2a). A comparison of the genomes of the LiPSC clones and LCLs revealed a deletion (233,645 bp) at 19 p13.3 in all the LiPSC clones examined from the healthy donor, KA (Fig. 2b-d). Although the number of reads was limited (approximately $6 \%$ of the total reads), the presence of the reads spanning the breakpoint was confirmed not only in the LiPSC clones but also in the LCLs (Fig. 2e), thus strongly suggesting that the deletions in the LiPSC clones were already present in a subpopulation of their original cells, LCLs and were not detected by the aCGH analysis.

We then searched for the appearance of SNVs by comparing the LiPSC clones with LCLs by using the WGS analysis. In this analysis, we focused on nonsynonymous SNVs in coding regions, and all the variations were further validated by direct nucleotide sequence analysis. The analysis revealed the appearance of 4-6 non-synonymous variations in the LiPSCs clones derived from KA. The analysis revealed the appearance of 9-12 non-synonymous SNVs in the PB-LiPSC clones compared with the original cell source, LCLs (Fig. 2a).

We identified a somatic mutation in SLC26A5 (rs758296903) in all the LiPSC clones from KA (LKA10, LKA29 and LKA36) compared with their original cells. A detailed examination of the short reads revealed that $8.6 \%$ of the reads from the LCLs carried the mutation, thus indicating that the SNV was already present in a subpopulation of the original cells. Other variations observed in the TiPSCs and DF-iPSCs compared with Tcells and DFs, respectively, are shown in Fig. 2a and Additional file 1: Figure S1. These data indicate that the reprogramming and/or immortalization processes might cause some somatic mutations. However, the total number of somatic mutations observed in the genomes of iPSCs compared with their corresponding original cells did not vary among the cell sources of origin (LCLs, Tcells and fibroblasts: Fig. 2a), thus suggesting that LiPSCs may also have the same properties and functions as hiPSCs, similarly to TiPSCs and DF-iPSCs.

\section{Differentiation of neural cells from LiPSCs through a direct neurosphere conversion method}

In our previous reports, we have shown that TiPSCs are poorly differentiated into the ectodermal lineage, and it is difficult to induce TiPSCs to differentiate into neuronal cells via EB formation by using spontaneous neuronal differentiation protocols. To overcome this limitation, we developed a neurosphere (NS)-based differentiation method (direct NS conversion method: dNS method). TiPSCs were differentiated into neural cells with similar efficiency as DF-iPSCs with the dNS method [6]. We used the dNS method to differentiate the LiPSCs into neural cells (Fig. 3a) because both TiPSCs and LiPSCs were derived from peripheral blood cells and supposedly have similar differentiation propensities. With the dNS method, LiPSCs efficiently formed similar number of NSs (Fig. 3b) compared with DFiPSCs (Fig. 3c). Morphological analysis of NSs focusing on their circularity (Fig. 3d) and diameter (Fig. 3e) also demonstrated that there were no significant differences between LiPSC- and DF-iPSC-NSs (Figs. 3d and e). We quantified the expression of pluripotent markers, NANOG and OCT4 and neural markers, PAX6 and NESTIN, in LiPSC-derived NSs by qPCR analysis. Although the expression of NANOG in LiPSC-derived NSs was lower in all clones and DF-iPSC-derived NSs (Fig. 3d), OCT4 expression was maintained in some LiPSCderived NS clones and DF-iPSC-derived NS clones (Fig. 3e). However, these differences in NANOG (Fig. 3h) and OCT4 expression (Fig. 3i) were not statistically significant among the cell types of origin. The expression of neural stem cell markers did not differ significantly among the various NSs we tested, regardless of the original cell type and donor (Fig. 3f, g, j and k). These results indicate that LiPSCs can differentiate into NSs via the dNS method as efficiently as DF-iPSCs.

\section{LiPSCs can be differentiated into various types of functional neurons to a similar level as DF-iPSCs by using the dNS method}

We next examined whether LiPSC-derived NSs could be induced to differentiate into neural cells as efficiently as DF-iPSCs. LiPSC-derived NSs were dissociated into single cells and cultured for neural differentiation. Thirteen days later, the differentiated cells were immunostained 


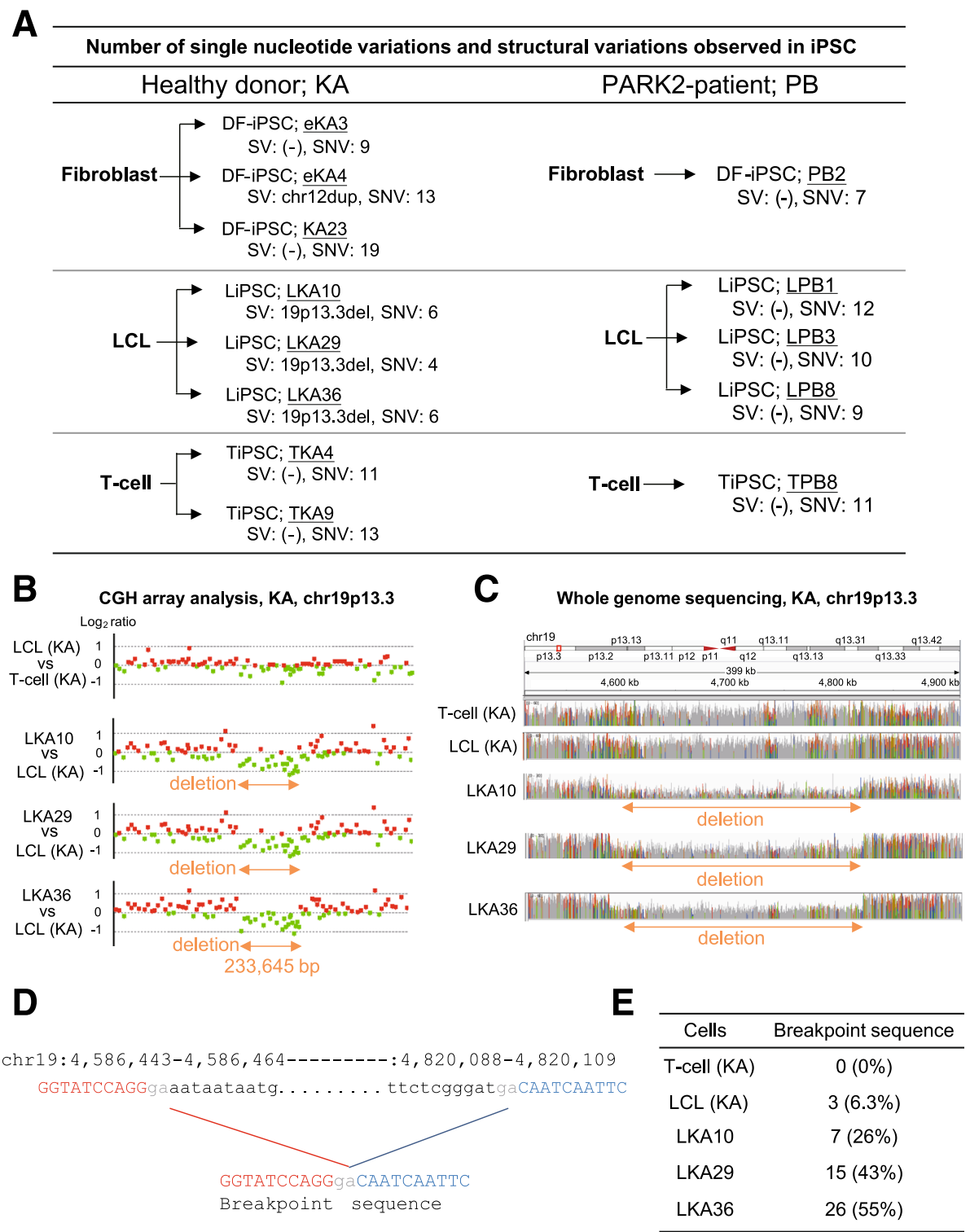

Fig. 2 De novo mutations and structural variations caused by the reprogramming process. a Summary of the number of somatic mutations. SVs were detected by an aCGH analysis. Candidate SNVs were identified by whole genome analysis and confirmed by a direct nucleotide sequence analysis. Only the nonsynonymous variants in protein coding regions outside the immunoglobulin or T-cell receptor gene regions are shown. b A recurrent structural variation in the short arm of chromosome 19 in healthy donor KA detected by CGH analysis is shown. The deletion was detected in all the LiPSC clones examined, but was not detected in the LCLs. c The coverage data for each sample (LKA10, LKA29 and LKA36), as shown by the Integrative Genomic Viewer [44]. In the LiPSC clones, decreased coverages were observed and corresponded to the deletions detected by the CGH analysis. $\mathbf{d}$ The breakpoint sequences were identified in the short reads obtained from the LCLs. Identical breakpoint sequences were also identified in the short reads obtained from LKA10, LKA29 and LKA36. There is a 2 bp microhomology at the breakpoint. e The short read sequences indicating the breakpoint were identified in only $6.3 \%$ of the total reads spanning this region in LCLs. Short read sequences indicating the same breakpoint were identified in all the LiPSC clones. These short read sequences were observed in 26,43 and $55 \%$ of the total reads obtained from LKA10, LKA29 and LKA36 clones, respectively

with antibodies against a neural marker, $\beta$ III-TUBULIN and an astrocyte marker, glial fibrillary acid protein (GFAP) (Fig. 4a). LiPSC-derived NSs differentiated into neurons and astrocytes at a similar ratio as DF-iPSCs, indicating that LiPSC-derived NSs had similar propensities to differentiate into neurons and astrocytes as the DF-
iPSC-derived NSs (Fig. 4b-d). The immunocytochemical analysis revealed that these BIII-TUBULIN-positive neurons included tyrosine hydroxylase (TH)-positive dopaminergic neurons (Fig. 4e and f), gammaaminobutyric acid (GABA)-positive GABAergic neurons (Fig. 4e and g), and vesicular glutamate transporter 1- 


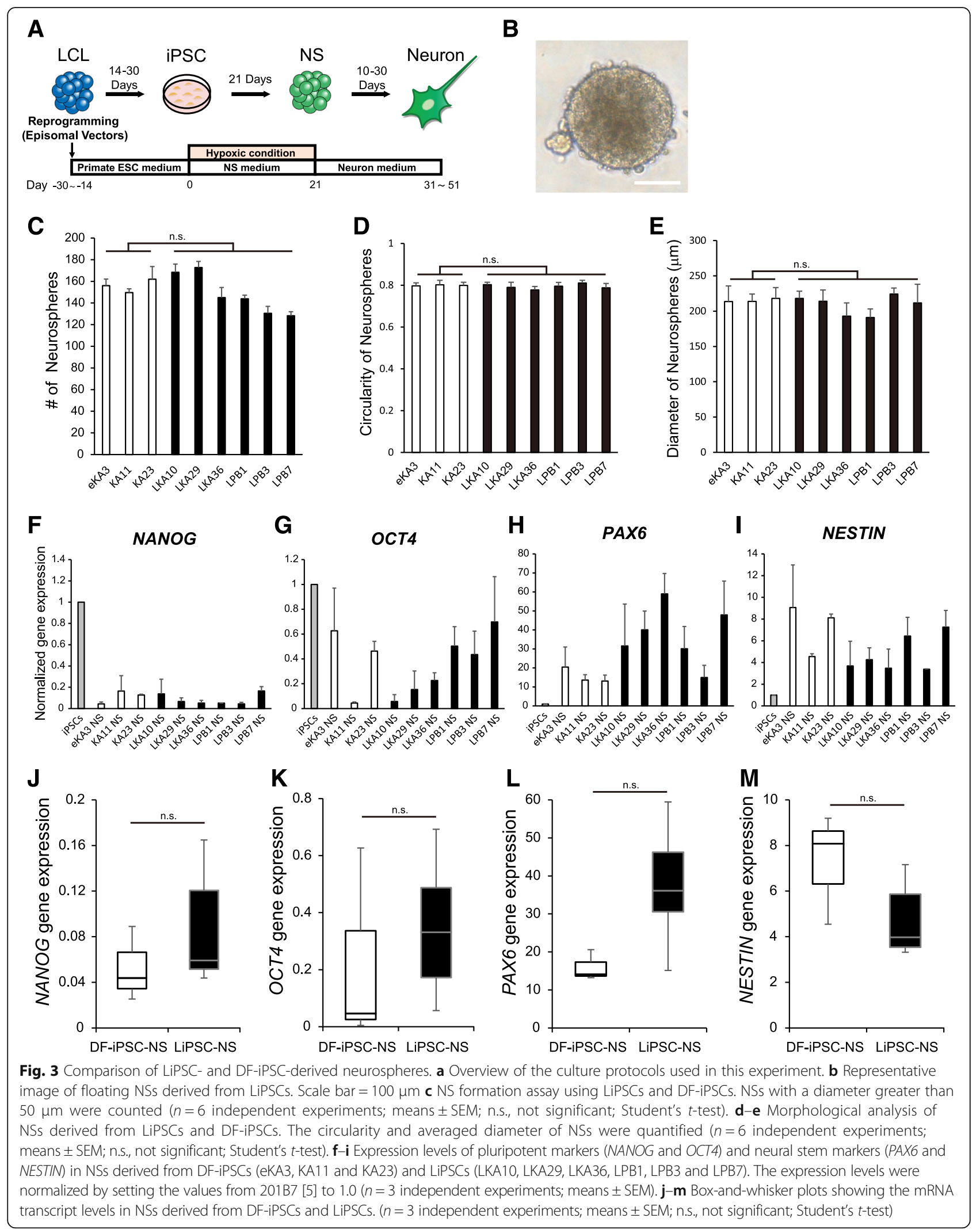




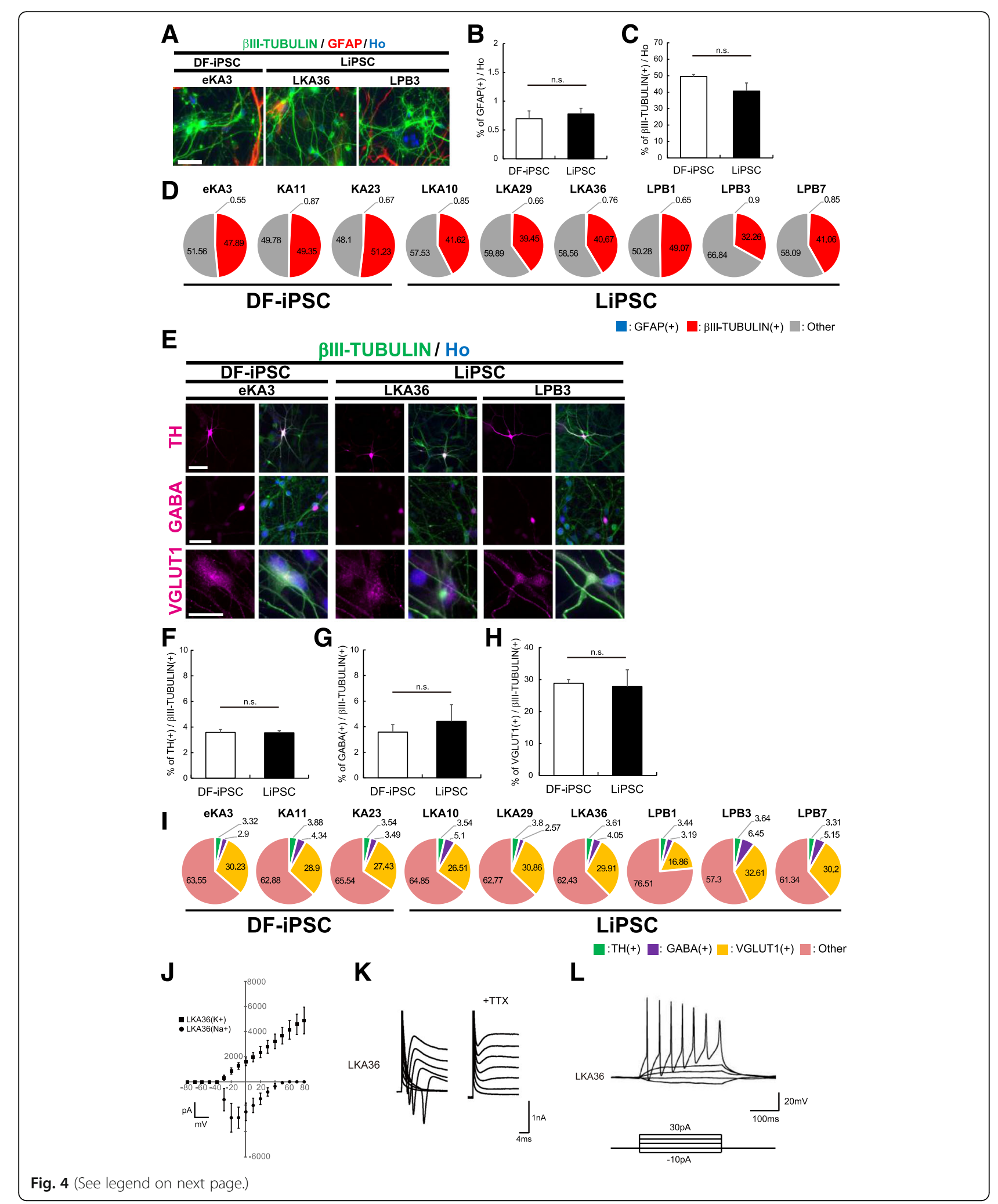


(See figure on previous page.)

Fig. 4 Generation of various types of functional neurons from LiPSCS with the dNS method. a Representative images of DF-iPSC- and LiPSC-derived neurons and glial cells stained with antibodies against the indicated markers. Scale bars $=50 \mu \mathrm{m}$. $\mathbf{b}-\mathbf{c}$ Ratio of cell types differentiated from DF-iPSCs and LiPSCs ( $n=3$ independent experiments; means \pm SEM; n.S., not significant; Student's $t$-test). $\mathbf{d}$ Summary of cell types produced from each hiPSC line $(n=3)$. e Representative images of DF-iPSC- and LiPSC-derived neuronal subtypes stained with antibodies against the

indicated markers. Scale bars $=50 \mu \mathrm{m}$. $\mathbf{f}-\mathbf{h}$ Ratio of neuronal subtypes differentiated from DF-iPSCs and LiPSCs $(n=3$ independent experiments; means \pm SEM; n.S., not significant; Student's t-test). i Summary of neuronal subtypes produced from each hiPSC line $(n=3)$. j Current-voltage plot of the sodium and potassium currents of neurons derived from LiPSCS ( $n=6$ independent experiments; means \pm SEM). $\mathbf{k}$ Voltage-dependent sodium and potassium currents in neurons derived from LiPSCs. I Representative traces of the membrane potential of LiPSC-derived neurons in response to step depolarization by a current injection

positive glutamatergic neurons (Fig. 4e and h). Figs. 4d and i show a summary of the data from these differentiated cell types and neuronal subtypes, suggesting that LiPSCs differentiated into various types of cells as efficiently as DF-iPSCs, regardless of the differences among donors.

We recorded voltage-sensitive currents in 60-dayold LiPSC-derived neurons to confirm their electrophysiological properties. LiPSC-derived NSs were infected with a lentivirus expressing a human Synap$\sin I$ promoter-driven GFP (CSIV-hSynI-GFP-IRES2NeoR) soon after single dissociated NS cells were plated [27]. After neuronal maturation, voltagedependent $\mathrm{Na}^{+}$and $\mathrm{K}^{+}$currents and TTX-sensitive voltage-gated membrane currents were detected (Fig. $4 \mathrm{j}$ and $\mathrm{k}$ ), and action potentials were elicited in the majority of the LiPSC-derived neuronal cells analyzed by depolarizing the membrane in current clamp mode (Fig. 41). These results suggested that LiPSCs can be differentiated into functional neurons through the dNS method.

\section{Neurons derived from PARK2-LiPSCs exhibited impaired mitochondrial activity and phenotypes}

We differentiated LiPSCs derived from a patient with parkin mutations, a familial form of Parkinson's disease, PARK2, into neurons to determine whether hiPSCs established from LCLs could be used as a model of neurological disease. A consistent neurochemical abnormality found in Parkinson's disease is the degeneration of dopaminergic neurons in the substantia nigra. Therefore, we exposed single dissociated hiPSCs to FGF8, sonic hedgehog (Shh), purmorphamine (PMA) and CHIR99021 during the NS formation, as described in our previous study [6], to generate midbrain dopaminergic neuron (mDAN)-enriched culture. After neuronal maturation for 13 days in culture, immunostaining of the neural epithelial cells demonstrated that nearly $20 \%$ of the BIII-TUBULIN-positive cells expressed the dopaminergic neuron marker $\mathrm{TH}$, with a similar differentiation ratio between the DF-iPSCs and LiPSCs (Fig. 5b and c). These data indicate that the LiPSCs and DFiPSCs were able to differentiate into mDANs with similar efficiencies (Fig. 5a-c).
A PCR analysis of the genomic DNA confirmed that PARK2 patient-derived LiPSCs (LPBs; LPB1, LPB3 and LPB7) contained a homozygous deletion in exons 6 and 7 of the parkin gene (Fig. 5d), which encodes a component of an E3 ubiquitin ligase involved in mitochondrial homeostasis [28]. We have previously reported that hiPSCs established from DFs of a PARK2 patient exhibited abnormal turnover of damaged mitochondria [29] and increased production of reactive oxygen species (ROS) in their neurons. Therefore, we treated the LiPSC-derived neurons with carbonyl cyanide m-chlorophenyl hydrazone (CCCP), which triggers the loss of mitochondrial membrane potential and results in the removal of damaged mitochondria. We visualized the area of the inner mitochondrial membrane (IMM) by using an antibody against the IMM marker Complex-III Core I (C-III Core I) to determine the extent to which the damaged mitochondria were eliminated after CCCP treatment. Compared with the untreated samples, the CCCP-treated samples elicited a dramatic decrease in the IMM area in the control neurons (LKA clones), but not in the PARK2 neurons (LPB clones) (Fig. 5e and f). Using the same CCCP-treated neuronal samples, we performed immunostaining with an anti-TH antibody to quantify the ratio of $\mathrm{TH}$-positive dopaminergic neurons. In both the DF-iPSC- and LiPSC-derived neurons, we observed significant decreases in the numbers of $\mathrm{TH}$ positive neurons in PARK2-derived cells (LPB clones) after the CCCP treatment, indicating that dopaminergic neurons derived from PARK2-iPSCs were more vulnerable to mitochondrial stress than control-iPSCs (Fig. 5g).

Finally, we evaluated ROS production in the neurons derived from the control- and PARK2-LiPSCs by using the CellROX ${ }^{\odot}$ Green Reagent, which is weakly fluorescent in a reduced state and exhibits bright green photostable fluorescence after oxidation by ROS, with absorption/emission maxima of $\sim 485 / 520 \mathrm{~nm}$. The reactive CellROX fluorescence was significantly increased in the PARK2 neurons, and this phenotype prominently appeared in the TH-positive neurons, indicating that ROS production was increased in the PARK2 neurons, particularly the mDANs (Fig. $5 \mathrm{~h}$ and i). These phenotypes were observed in both DF-iPSC- and LiPSCderived neurons (Figs. 5e-i). These results strongly 


\section{A}

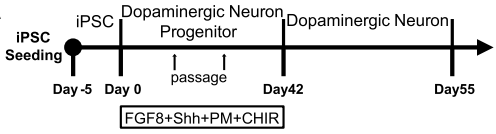

B

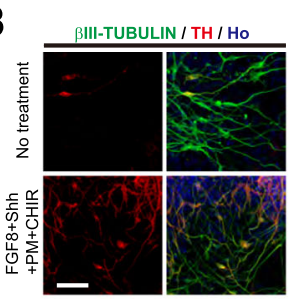

\section{C}
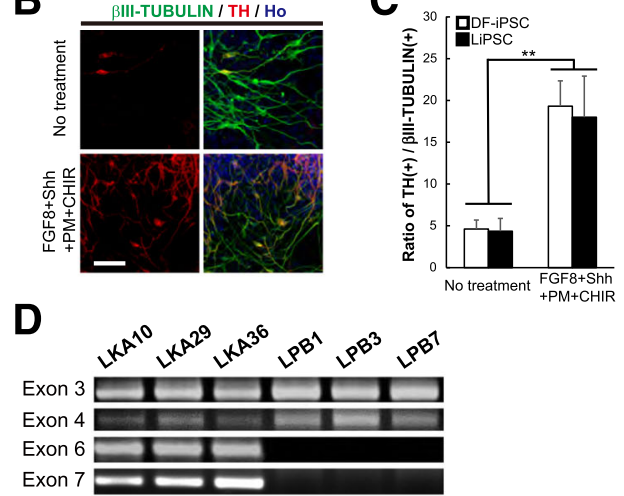

E

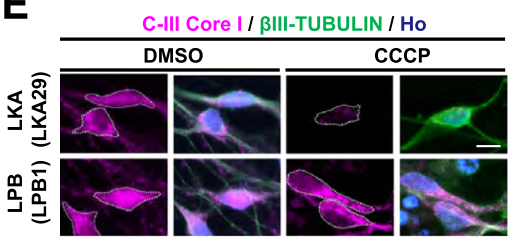

F
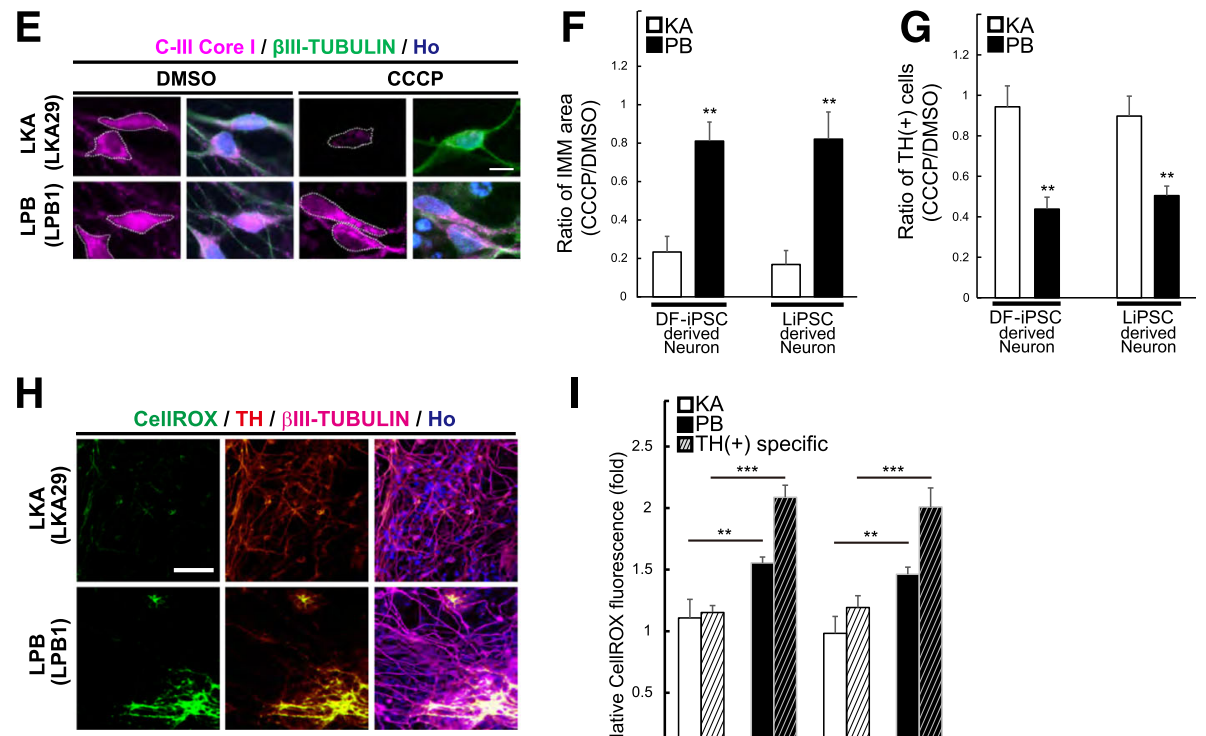

I

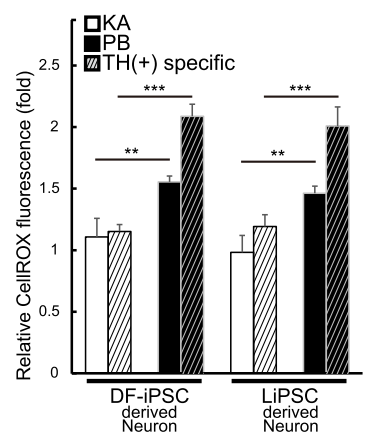

Fig. 5 Reproduction of Parkinson's disease phenotypes using neurons derived from PARK2-LiPSCS. a An overview of the culture protocol used in this experiment. $\mathbf{b}$ Immunostaining of LiPSC (LPB10)-derived dopaminergic neurons with antibodies raised against the indicated markers. Scale bars $=100 \mu \mathrm{m}$. $\mathbf{c}$ Analysis of dopaminergic neuron differentiation by quantifying BIII-TUBULIN and TH double-positive neurons at Day 55 ( $n=3$ independent experiments; means \pm SEM; ${ }^{* *} p<0.01$; Student's $t$-test). $\mathbf{d}$ The deletion of exons 6 and 7 was confirmed in clones LPB1, LPB3 and LPB7. e Double labeling for the IMM marker CIII-Core I and BIII-TUBULIN in DMSO- or CCCP-treated DF-iPSC- and LiPSC-derived neurons from a healthy donor or a PARK2 patient. Scale bar $=50 \mu \mathrm{m}$. $\mathbf{f}$ The ratio of the IMM area was determined by quantifying the CCCP/DMSO ratio of CIII-Core I staining in the BIII-TUBULIN-positive cells ( $n=3$ independent experiments; means \pm SEM; ${ }^{* *} p<0.01$; Student's $t$-test). $\mathbf{g}$ The CCCP treatment significantly decreased the ratio of TH-positive neurons in the PARK2 patient-derived neurons compared with the control ( $n=3$ independent experiments; means \pm SEM; ${ }^{* *} p<0.01$; Student's $t$-test). $\mathbf{h}$, i Analysis of oxidative stress using the CellROX ${ }^{\circledR}$ Green Reagent. The PARK2 neurons showed increased CellROX fluorescence compared with the control neurons ( $n=3$ independent experiments; means \pm SEM; ${ }^{* *} p<0.01,{ }^{* * *} p<0.001$; Student's t-test). Scale bars $=500 \mu \mathrm{m}$

suggest that the hiPSCs derived from patient LCLs can be used as models of neurological diseases.

\section{Discussion}

Patient-specific iPSC technology makes it possible to recapitulate disease phenotypes in vitro, thus significantly facilitating the elucidation of disease processes and the development of therapeutic drugs. However, invasive skin biopsies are generally performed to obtain a patient's original cells, DFs and generate iPSCs. Several groups have succeeded in establishing iPSCs from peripheral blood cells, T-cells and LCLs, thus extending 
the use of iPSC technologies [15-19, 30, 31]. In particular, our previous study has demonstrated the utility of TiPSCs as tools for modeling diseases. However, it had been unclear whether LiPSCs can function as a disease model. Because LCLs are stable cell lines that are easy to culture, they are expected to be the ideal resource for efficient wide-scale parallel reprogramming to achieve high-throughput processing for modeling polygenic or sporadic diseases. Moreover, various types of LCL clones established from patients are already available and stored in global repositories [12]. Therefore, it would be very important to obtain experimental proof of the utility of LiPSCs as disease models to increase the number of cases, perform disease-specific analyses based on statistical analysis, and expand the target-disease areas for iPSC technology.

In addition to this study, several groups have established iPSCs from peripheral blood cells and have investigated their characteristics as iPSCs $[6,7,15-19,30$, 31]. However, little is known about the effects of the differences among original cells on genomic mutations in the derived iPSCs. In particular, LiPSCs are highly influenced by the original cells because LCLs are generated through an EBV infection-mediated immortalization process. This study clearly showed that the differences among LCLs compared with the original cells had no substantial effects on the number of somatic single nucleotide variations and structural variations in the established iPSCs compared with those obtained from T-cells or DFs (Fig. 2). These data broaden the applications of LiPSCs, and strongly support the utility of LCLs as original cells for generating iPSCs. Moreover, our results indicated that the reprogramming processes can cause some somatic mutations, regardless of the cell type of origin (Fig. 2 and Additional file 2: Figure S2). Although some of these mutations have recently been described [32], further studies are needed to clarify their effects.

In recent studies of human clinical genetics, progress has been made in the identification of the genes responsible for neurological disorders [33, 34] and the genes increasing the onset risk of neuropsychiatric disorders [35]. However, there are many diseases for which the causative genes and genetic risk factors have not yet been identified. Recent whole exome sequencing studies have revealed that these sporadic cases are related to many variants, indicating the cumulative genetic mutations that trigger the onset of the disease. It is important to establish hiPSCs from a sufficient number of patients and characterize multiple clones for statistical analyses to elucidate the pathogenic mechanisms of these diseases. Well-characterized LCLs are already available in global repositories and are linked to patient clinical history, long-term genotype and phenotype data, and molecular/functional studies. Then, the clarification of phenotypes using hiPSC disease model could elucidate the pathological mechanism of such sporadic diseases, and provide a path to the identification of their related genes. Therefore, in addition to T-cells, LCLs are a suitable source for generating hiPSCs to model sporadic or common diseases.

\section{Methods}

\section{Human iPSC generation from LCLs}

Peripheral blood mononuclear cells (PBMCs) were obtained from a healthy donor, "KA" and a patient with autosomal recessive juvenile Parkinson's disease (PARK2), "PB", by centrifuging heparinized blood over a Ficoll-Paque PREMIUM (GE Healthcare, Chicago, USA) gradient. KA- or PB-derived PBMCs were immortalized by an EBV infection according to the protocol of SRL Medisearch Incorporation and were transformed into LCLs. LCLs were cultured in RPMI 1640 (Gibco, Massachusetts, USA) supplemented with $10 \%$ fetal bovine serum (FBS) at $37{ }^{\circ} \mathrm{C}$ and $5 \% \mathrm{CO}_{2}$ in a humidified incubator. After several passages, the LCLs were electroporated with the $\mathrm{Neon}^{\mathrm{Tm}}$ Transfection System $100 \mu \mathrm{L}$ Kit (MPK10096; Thermo Fisher Scientific, Massachusetts, USA) using $1.0 \mu \mathrm{g}$ of each episomal plasmid (Addgene, Cambridge, USA) expressing 6 factors: OCT4, SOX2, KLF4, l-MYC, LIN28 and p53 shRNAs (pCXLE-hOCT3/4-shp53, pCXLE-hSK and pCXLE-hUL), according to the manufacturer's instructions. The transfected LCLs were rapidly transferred to a 6-well plate at a density of $2.0 \times 10^{6}$ cells/well and incubated for $24 \mathrm{~h}$. At $24 \mathrm{~h}$ after electroporation, the medium was replaced with hiPSC medium. After an additional $24 \mathrm{~h}$, the cells were passaged to a $100 \mathrm{~mm}$ dish containing mitomycin C-inactivated mouse SNL feeder cells at a density of $5.0 \times 10^{4}$ $5.0 \times 10^{5}$ cells/dish and cultured in hiPSC medium, which was changed every other day until colonies were picked. The generated hiPSCs were maintained on mitomycin Cinactivated mouse SNL feeder cells in hiPSC medium. All hiPSC lines analyzed in this study were between passage 7 and 20.

All human primary cells were used after appropriate written informed consent was given to the commercial providers. All experimental procedures for biopsy and reprogramming were approved by the Ethics Committee of the Keio University School of Medicine (No. 20080016).

\section{Neural differentiation in vitro}

The dNS method, which we have previously reported, was used for the neural differentiation of hiPSCs [6]. For neural induction, hiPSCs were dissociated into single cells by incubation with TrypLE $^{\mathrm{TM}}$ Select (Life Technologies, Massachusetts, USA) for $5 \mathrm{~min}$ and 
pipetting. The cells were cultured at a density of 10 cells $/ \mu \mathrm{L}$ in a T25 flask (Nunclon, Massachusetts, USA) in MHM supplemented with B27 (Gibco, Massachusetts, USA), $20 \mathrm{ng} / \mathrm{mL}$ FGF-2 (Wako, Osaka, Japan), $10 \mu \mathrm{M}$ Y-27632 (Wako, Osaka, Japan) and $10 \mathrm{ng} / \mathrm{mL}$ hLIF (Nacalai Tesque, Kyoto, Japan) in $4 \%$ oxygen for 7 days. NSs were repeatedly passaged by dissociation into single cells, and then cultured at a density of 50 cells $/ \mu \mathrm{L}$ in the same manner as the primary sphere formation. NSs were used at passage 3 for analysis. For terminal differentiation, the dissociated NSs were plated on PO- (SigmaAldrich, Missouri, USA) and fibronectin- (Sigma-Aldrich, Missouri, USA) coated coverslips and cultured in MHM containing B27 (Gibco, Massachusetts, USA), $10 \mathrm{ng} / \mathrm{mL}$ brain-derived neurotrophic factor (BDNF; R\&D Systems, Minnesota, USA), $10 \mathrm{ng} / \mathrm{mL}$ glial celldifferentiated neurotrophic factor (GDNF; R\&D Systems, Minnesota, USA), $200 \mu \mathrm{M}$ ascorbic acid (Sigma-Aldrich, Missouri, USA) and $1 \mathrm{mM}$ dibutyryl-cAMP (Sigma-Aldrich, Missouri, USA) for 10-60 days.

\section{Immunocytochemical analysis of hiPSCs and neurons derived from hiPSCs}

Cells were fixed in phosphate-buffered saline (PBS) containing $4 \%$ paraformaldehyde (PFA) for $30 \mathrm{~min}$ at room temperature. Thereafter, all cells were blocked with $5 \%$ FBS and Triton X-100 and incubated with the primary antibodies described in Additional file 3: Table S1. The cells were then rinsed with PBS, and incubated with species-specific Alexa Fluor 488-, Alexa Fluor 555- or Alexa Fluor 647-conjugated secondary antibodies (1:500; Invitrogen, Massachusetts, USA); this was followed by Hoechst 33258 (0.5 $\mu \mathrm{g} / \mathrm{mL}$; Sigma-Aldrich, Missouri, USA) to counterstain the nuclei. The images were obtained using a universal fluorescence microscope (Axioplan2; Carl Zeiss AG, Oberkochen, Germany) or a confocal laser scanning microscope (LSM-710; Carl Zeiss AG, Oberkochen, Germany).

\section{CGH array analysis}

Genomic DNA was extracted using a DNeasy Blood \& Tissue Kit (QIAGEN, Hilden, Germany) according to the manufacturer's protocol. Five hundred micrograms of genomic DNA was subjected to the Human CGH array (4X180K; Agilent Technologies, California, USA) according to the manufacturer's protocol. All combinations (original cells and iPSCs) were analyzed. The data were analyzed using the Agilent Genomic Workbench. Rearrangements involving immunoglobulin gene regions and $\mathrm{T}$-cell receptor gene regions that are rearranged in $\mathrm{T}$-cell and $\mathrm{B}$-cell lineages, respectively, were not taken into consideration in the analysis of the structural variations.

\section{Whole genome sequence analysis}

One microgram of genomic DNA was subjected to whole genome sequence analysis. Sequencing libraries were constructed using a TruSeq DNA PCR-free library preparation kit (Illumina, California, USA). The sequences were analyzed using HiSeq2500 (Illumina, California, USA) in the rapid mode (150 bp, paired end). After alignment to the reference genome (hg19) using BWA [36] and removal of multiply aligned reads and duplicate reads, basecalls were performed with SAMtools [37]. Somatic variants were detected as previously described [38, 39]. We confirmed that all of the candidate somatic mutations consisting of single nucleotide substitutions were predicted to result in changes in the amino acid sequence by direct nucleotide sequence analysis. The SNVs inside T-cell receptor regions and immunoglobulin regions were excluded. If necessary, whole genome amplification using REPLI-g (QIAGEN, Hilden, Germany) was applied to the direct nucleotide sequence analysis.

\section{Microarray analysis}

Total RNA was extracted with an RNAeasy Kit (QIAGEN, Hilden, Germany) and the RNA quality was assessed using an Agilent 2100 Bioanalyzer (Agilent Technologies, California, USA). Total RNA (100 ng) was reverse-transcribed, labeled with biotin using a 3'IVT Express Kit (Affymetrix, California, USA) and hybridized to a GeneChip ${ }^{\oplus}$ Human Genome U133 plus 2.0 Array (Affymetrix, California, USA). The arrays were washed and stained using a GeneChip Fluidics Station 450 (Affymetrix, California, USA) and then scanned with the GeneChip Scanner 3000 7G System (Affymetrix, California, USA) according to the manufacturer's instructions. The raw probe intensity files were MAS5normalized and log (base2) transformed by using GeneSpring GX 13.1 software (Agilent Technologies, California, USA). The gene set was filtered based on the expression levels to remove the genes that were not expressed in all samples. Principal component analysis (PCA) was performed using the normalized data. For the hierarchical clustering, the normalized data were calculated on the basis of Euclidean correlations with average linkages.

We used NCBI GEO microarray data GSE76832 for Tcell, DF and iPSCs established from them (T-cell(KA), DF(KA), TKA4, TKA9, eKA3 and KA11) [6].

\section{Reverse-transcription PCR}

Total RNA was isolated using an RNeasy Mini Kit (QIAGEN, Hilden, Germany) and $1 \mu \mathrm{g}$ of RNA was used to generate cDNAs by using a reverse transcription (RT) system (Promega, Wisconsin, USA). RT-PCR was performed as previously described [40]. Values were 
normalized to $A C T B$. Quantitative RT-PCR (qPCR) was performed on an ABI PRISM Sequence Detection System 7900HT (Applied BioSystems, Massachusetts, USA) by using SYBR premix ExTaq Tli RNaseH Plus (Takara Bio, Shiga, Japan). The primers are described in Additional file 4: Table S2.

\section{PCR amplification of genomic DNA}

Genomic DNA was purified from HDFs, LCLs and hiPSCs using a DNeasy Kit (QIAGEN, Hilden, Germany). The PCR conditions were described previously (Additional file 4: Table S2) [41].

\section{Electrophysiological analysis}

For the electrophysiology experiments, the culture medium was replaced with a physiological solution $(118 \mathrm{mM} \mathrm{NaCl}, 2.5 \mathrm{mM} \mathrm{KCl}, 26 \mathrm{mM} \mathrm{NaHCO}, 1 \mathrm{mM}$ $\mathrm{NaH}_{2} \mathrm{PO}_{4}, 10 \mathrm{mM}$ glucose, $4 \mathrm{mM} \mathrm{MgCl}$, and $4 \mathrm{mM}$ $\mathrm{CaCl}_{2}$ ). Tetrodotoxin (TTX, $1 \mu \mathrm{M}$ ) was bath-applied. The electrodes (5-8 M $\Omega$ ) were filled with whole-cell pipette solution $(120 \mathrm{mM}$ potassium acetate, $20 \mathrm{mM}$ $\mathrm{KCl}, 0.1 \mathrm{mM} \mathrm{CaCl}, 5 \mathrm{mM} \mathrm{MgCl}_{2}, 0.2 \mathrm{mM}$ EGTA, $5 \mathrm{mM}$ ATP, and $10 \mathrm{mM}$ HEPES, $\mathrm{pH}$ 7.3) [42, 43]. The whole-cell recordings of GFP-expressing neurons were configured using an EPC-7 amplifier (HEKA Elektronik Dr. Schulze GmbH, Lambrecht/Pfalz, Germany) and a Digidata 1200 acquisition board (Axon Instruments, California, USA). The membrane potential was clamped at $-60 \mathrm{mV}$. Membrane resistance $(R \mathrm{~m})$, series resistance $(R \mathrm{~s})$ and membrane capacitance $(\mathrm{Cm})$ were monitored. Only recordings with $R \mathrm{~m}>100 \mathrm{M} \Omega$ and $R \mathrm{~s}<20 \mathrm{M} \Omega$ were included in the analysis.

\section{Carbonyl cyanide m-chlorophenylhydrazone treatment}

Neurons were cultured with $30 \mu \mathrm{M}$ carbonyl cyanide m-chlorophenyl hydrazone (CCCP; Sigma-Aldrich, Missouri, USA) or DMSO for $48 \mathrm{~h}$. The cells were then fixed, stained for BIII-TUBULIN and ComplexIII Core I (CIII-Core I), and counterstained with Hoechst. The cytoplasmic area was extracted to quantify the IMM area of neurons, as shown in Fig. 5e. The IMM area of the neurons was quantified from the digitized values using IN CELL Analyzer 6000 (GE Healthcare, Chicago, USA).

\section{Oxidative stress analysis}

The ROS levels were determined by measuring the CellROX fluorescence using the CellROX ${ }^{\bullet}$ Green Reagent to detect oxidative stress (Life Technologies, Massachusetts, USA). Briefly, the neurons were incubated with the CellROX Reagent for $30 \mathrm{~min}$ at $37^{\circ} \mathrm{C}$, after which they were washed with PBS and then fixed with $4 \%$ PFA for $30 \mathrm{~min}$ at room temperature. Then, the cells were incubated with the primary antibody against $\beta$ III-TUBULIN (1:1000; Sigma-Aldrich, Missouri, USA) overnight at $4{ }^{\circ} \mathrm{C}$, washed with PBS and incubated with an Alexa Fluor 555-conjugated secondary antibody (1:500; Invitrogen, Massachusetts, USA) for $1 \mathrm{~h}$ at room temperature. The fluorescence of the $\beta$ III-TUBULIN-positive neurons was measured by using an IN Cell Analyzer 6000 (GE Healthcare, Chicago, USA).

\section{Additional files}

Additional file 1: Figure S1. In vitro differentiation analysis and karyotyping of LiPSC. (A) Representative karyotype of the established iPSC line from LCL-PB. (B) Quantitative analysis of immunocytochemistry for markers of three-germ layers; $\beta I I I-T U B U L I N$ (ectoderm), aSMA (mesoderm) and AFP (endoderm) based on their fluorescence intensities ( $n=3$ independent experiments; means \pm SEM; n.S., not significant; ANOVA). (C) Representative images of immunocytochemistry for the in vitro three-germ layer markers ( $\beta I I I-T U B U L I N$, aSMA and AFP). Scale bars $=50 \mu \mathrm{m}$. (PDF $500 \mathrm{~kb}$ )

Additional file 2: Figure S2. Somatic mutations in TiPSCs caused by the reprogramming process. a A substantial number of nonsynonymous variants were suggested to disappear in TKA4, all of which are located on chromosome 9, and Sanger sequencing showed an apparent mosaic loss of heterozygosity. An example of the Sanger sequence analysis of apparently lost nonsynonymous variant is shown. A small peak indicating a variant was observed in TKA4, suggesting a mosaic loss of heterozygosity. b The CGH analysis of TKA4 compared with the T-cells showed there were no copy number alterations in chromosome 9. However, a detailed inspection of the ratios of variants/reference bases revealed that the ratios were significantly different from 0.5 in the long arm of chromosome 9 of TKA4, supporting mosaic loss of heterozygosity in the region. Because the $\mathrm{CGH}$ analysis did not support copy number alterations (upper panel), these findings are more likely to represent somatic parental disomy involving the long arm of chromosome 9. A similar observation has recently been described [39]. (PDF 580 kb)

Additional file 3: Table S1. List of antibodies. (PDF $400 \mathrm{~kb}$ )

Additional file 4: Table S2. List of primers. (PDF 397 kb)

\section{Abbreviations}

CGH: Comparative genomic hybridization; DF: Dermal fibroblasts; DF-iPSC: DF-derived iPSC; dNS method: Direct neurosphere conversion method; EBV: Epstein-Barr virus; iPSC: Induced pluripotent stem cells; LCL: Lymphoblastoid B-cell lines; LiPSC: LCL-derived iPSC; mDAN: Midbrain dopaminergic neuron; NS: Neurosphere; ROS: Reactive oxygen species; SNV: Single nucleotide variation; SV: Structural variation; TH: Tyrosine hydroxylase; TiPSC: T-cell derived iPSC; WGS: Whole genome sequencing

\section{Acknowledgements}

The authors thank all of the members of H.O.'s laboratory for their encouragement and support.

\section{Funding}

This work was supported by funding from the Project for the Realization of Regenerative Medicine and Support for Core Institutes for iPS Cell Research from the Ministry of Education, Culture, Sports, Science and Technology of Japan (MEXT) to H.O., the Research Center Network for Realization Research Centers/Projects of Regenerative Medicine (the Program for Intractable Disease Research utilizing disease-specific iPS (ells) from the Japan Science and Technology Agency (JST) and Japan Agency for Medical Research and Development (AMED) to W.A. and H.O., the New Energy and Industrial Technology Development Organization (NEDO) to W.A. and H.O., the Japan Society for the Promotion of Science (JSPS) to W.A., and a Grant-in-Aid for the Global COE Program from MEXT to Keio University. 


\section{Availability of data and materials}

The data discussed in this publication have been deposited in the NCBI Gene Expression Omnibus (GEO, http://www.ncbi.nlm.nih.gov/geo/) database and are accessible through GEO Series accession numbers GSE76832 [6] and GSE82159.

\section{Author's contributions}

$\mathrm{KF}, \mathrm{TT}, \mathrm{TM}, \mathrm{RH}, \mathrm{ST}, \mathrm{WA}$ and $\mathrm{HO}$ conceived and designed the experiments. $\mathrm{KF}, T \mathrm{Te}, \mathrm{HI}, \mathrm{JM}, \mathrm{KD}$, JY, HT, TM, MI, TTa, ST and WA performed the experiments and analyzed data. KF, TTe, HI, JM, KD, JY, ST, WA and $\mathrm{HO}$ wrote and edited the manuscript. $\mathrm{RH}, \mathrm{NH}, \mathrm{SM}$ and TTa contributed reagents, materials and analysis tools. All authors read and approved the final manuscript.

\section{Competing interests}

H.O. is a paid Scientific Advisory Board Member of SanBio Co., Ltd. T.M. is employed by Ajinomoto Co., Inc. M.I. is employed by Sumitomo Dainippon Pharma Co., Ltd.1

\section{Consent for publication}

Written informed consent to publish was obtained from the patient and the healthy donor

\section{Ethics approval and consent to participate}

All human primary cells were used after appropriate written informed consent was given to the commercial providers. All experimental procedures for biopsy and reprogramming were approved by the Ethics Committee of the Keio University School of Medicine (No. 20080016).

\section{Author details}

'Department of Physiology, Keio University, School of Medicine, Shinjuku-ku, Tokyo 160-8582, Japan. 'Department of Neurology, Graduate School of Medicine, The University of Tokyo, Bunkyo-ku, Tokyo 113-8655, Japan. ${ }^{3}$ Department of Computational Biology and Medical Sciences, Graduate School of Frontier Sciences, The University of Tokyo, Kashiwa 277-0882, Japan. ${ }^{4}$ Department of Physiology, Yokohama City University Graduate School of Medicine, Kanazawa-ku, Kanagawa 236-0027, Japan. ${ }^{5}$ Department of Integrative Aging Neuroscience, Section of Neuroendocrinology, National Center for Geriatrics and Gerontology, Obu, Aichi 474-8511, Japan. ${ }^{6}$ Institute for Innovation, Ajinomoto Co., Inc., Kawasaki-ku, Kanagawa 210-8681, Japan. ${ }^{7}$ Molecular Research Center for Children's Mental Development, United Graduate School of Child Development, Osaka University, Suita-shi, Osaka 565-0871, Japan. ${ }^{8}$ Department of Psychiatry, Osaka University Graduate School of Medicine, Suita-shi, Osaka 565-0871, Japan. ${ }^{9}$ Department of Neurology, Juntendo University, School of Medicine, Bunkyo-ku, Tokyo 113-8431, Japan. ${ }^{10}$ Medical Genome Center, The University of Tokyo Hospital, Bunkyo-ku, Tokyo 113-8655, Japan. ${ }^{11}$ Center for Genomic and Regenerative Medicine, Juntendo University, School of Medicine, Bunkyo-ku, Tokyo 113-8431, Japan

\section{Received: 27 June 2016 Accepted: 20 September 2016} Published online: 03 October 2016

\section{References}

1. Imaizumi Y, Okano H. Modeling human neurological disorders with induced pluripotent stem cells. J Neurochem. 2014;129(3):388-99.

2. Marchetto MC, Gage FH. Modeling brain disease in a dish: really? Cell Stem Cell. 2012;10(6):642-5.

3. Mattis VB, Svendsen CN. Induced pluripotent stem cells: a new revolution for clinical neurology? Lancet Neurol. 2011;10(4):383-94.

4. Okano H, Yamanaka S. iPS cell technologies: significance and applications to CNS regeneration and disease. Mol Brain. 2014;7:22.

5. Takahashi K, Tanabe K, Ohnuki M, Narita M, Ichisaka T, Tomoda K, Yamanaka S. Induction of pluripotent stem cells from adult human fibroblasts by defined factors. Cell. 2007;131(5):861-72

6. Matsumoto T, Fujimori K, Andoh-Noda T, Ando T, Kuzumaki N, Toyoshima M, Tada H, Imaizumi K, Ishikawa M, Yamaguchi R, et al. Functional Neurons Generated from T Cell-Derived Induced Pluripotent Stem Cells for Neurological Disease Modeling. Stem Cell Rep. 2016;6(3):422-35.
7. Seki T, Yuasa S, Fukuda K. Generation of induced pluripotent stem cells from a small amount of human peripheral blood using a combination of activated T cells and Sendai virus. Nat Protoc. 2012;7(4):718-28.

8. Klebanoff CA, Gattinoni L, Restifo NP. CD8+ T-cell memory in tumor immunology and immunotherapy. Immunol Rev. 2006;211:214-24.

9. Nishimura T, Kaneko S, Kawana-Tachikawa A, Tajima Y, Goto H, Zhu D, Nakayama-Hosoya K, Iriguchi S, Uemura Y, Shimizu T, et al. Generation of rejuvenated antigen-specific $T$ cells by reprogramming to pluripotency and redifferentiation. Cell Stem Cell. 2013;12(1):114-26.

10. Wherry EJ. T cell exhaustion. Nat Immunol. 2011;12(6):492-9.

11. Sie L, Loong S, Tan EK. Utility of lymphoblastoid cell lines. J Neurosci Res. 2009;87(9):1953-9.

12. Wheeler HE, Dolan ME. Lymphoblastoid cell lines in pharmacogenomic discovery and clinical translation. Pharmacogenomics. 2012;13(1):55-70.

13. Beck JC, Beiswanger CM, John EM, Satariano E, West D. Successful transformation of cryopreserved lymphocytes: a resource for epidemiological studies. Cancer Epidemiol Biomark Prev. 2001;10(5):551-4.

14. Worthington J, Ollier WE, Leach MK, Smith I, Hay EM, Thomson W, Pepper L, Carthy D, Farhan A, Martin S, et al. The Arthritis and Rheumatism Council's National Repository of Family Material: pedigrees from the first 100 rheumatoid arthritis families containing affected sibling pairs. Br J Rheumatol. 1994;33(10):970-6.

15. Barrett R, Ornelas L, Yeager N, Mandefro B, Sahabian A, Lenaeus L, Targan SR, Svendsen CN, Sareen D. Reliable generation of induced pluripotent stem cells from human lymphoblastoid cell lines. Stem Cells Transl Med. 2014;3(12):1429-34

16. Bueno C, Sardina JL, Di Stefano B, Romero-Moya D, Munoz-Lopez A, Ariza L, Chillon MC, Balanzategui A, Castano J, Herreros A, et al. Reprogramming human $B$ cells into induced pluripotent stem cells and its enhancement by C/EBPalpha. Leukemia. 2016:30(3):674-82.

17. Choi SM, Liu H, Chaudhari P, Kim Y, Cheng L, Feng J, Sharkis S, Ye Z, Jang YY. Reprogramming of EBV-immortalized B-lymphocyte cell lines into induced pluripotent stem cells. Blood. 2011;118(7):1801-5.

18. Rajesh D, Dickerson SJ, Yu J, Brown ME, Thomson JA, Seay NJ. Human lymphoblastoid B-cell lines reprogrammed to EBV-free induced pluripotent stem cells. Blood. 2011;118(7):1797-800.

19. Thomas SM, Kagan C, Pavlovic BJ, Burnett J, Patterson K, Pritchard JK, Gilad Y. Reprogramming LCLs to iPSCs Results in Recovery of DonorSpecific Gene Expression Signature. PLoS Genet. 2015;11(5):e1005216.

20. Amoli MM, Carthy D, Platt H, Ollier WE. EBV Immortalization of human B lymphocytes separated from small volumes of cryo-preserved whole blood. Int J Epidemiol. 2008;37 Suppl 1:i41-5.

21. Nilsson K. Human B-lymphoid cell lines. Hum Cell. 1992;5(1):25-41.

22. Sugimoto $M$, Tahara $H$, Ide T, Furuichi $Y$. Steps involved in immortalization and tumorigenesis in human B-lymphoblastoid cell lines transformed by Epstein-Barr virus. Cancer Res. 2004;64(10):3361-4.

23. Kim K, Doi A, Wen B, Ng K, Zhao R, Cahan P, Kim J, Aryee MJ, Ji H, Ehrlich LI, et al. Epigenetic memory in induced pluripotent stem cells. Nature. 2010:467(7313):285-90

24. Kim K, Zhao R, Doi A, Ng K, Unternaehrer J, Cahan P, Huo H, Loh YH, Aryee MJ, Lensch MW, et al. Donor cell type can influence the epigenome and differentiation potential of human induced pluripotent stem cells. Nat Biotechnol. 2011:29(12):1117-9.

25. Polo JM, Liu S, Figueroa ME, Kulalert W, Eminli S, Tan KY, Apostolou E, Stadtfeld M, Li Y, Shioda T, et al. Cell type of origin influences the molecular and functional properties of mouse induced pluripotent stem cells. Nat Biotechnol. 2010;28(8):848-55.

26. Altmann M, Pich D, Ruiss R, Wang J, Sugden B, Hammerschmidt W. Transcriptional activation by EBV nuclear antigen 1 is essential for the expression of EBV's transforming genes. Proc Natl Acad Sci U S A. 2006;103(38):14188-93.

27. Zhou Z, Kohda K, Ibata K, Kohyama J, Akamatsu W, Yuzaki M, Okano HJ, Sasaki E, Okano H. Reprogramming non-human primate somatic cells into functional neuronal cells by defined factors. Mol Brain. 2014;7:24.

28. Shimura H, Hattori N, Kubo S, Mizuno Y, Asakawa S, Minoshima S, Shimizu N, Iwai K, Chiba T, Tanaka K, et al. Familial Parkinson disease gene product, parkin, is a ubiquitin-protein ligase. Nat Genet. 2000:25(3):302-5

29. Imaizumi Y, Okada Y, Akamatsu W, Koike M, Kuzumaki N, Hayakawa H, Nihira T, Kobayashi T, Ohyama M, Sato S, et al. Mitochondrial dysfunction associated with increased oxidative stress and alpha-synuclein accumulation 
in PARK2 IPSC-derived neurons and postmortem brain tissue. Mol Brain. 2012;5:35.

30. Loh YH, Hartung O, Li H, Guo C, Sahalie JM, Manos PD, Urbach A, Heffner GC, Grskovic M, Vigneault F, et al. Reprogramming of T cells from human peripheral blood. Cell Stem Cell. 2010;7(1):15-9.

31. Seki T, Yuasa S, Oda M, Egashira T, Yae K, Kusumoto D, Nakata H, Tohyama $\mathrm{S}$, Hashimoto H, Kodaira M, et al. Generation of induced pluripotent stem cells from human terminally differentiated circulating T cells. Cell Stem Cell. 2010;7(1):11-4

32. Okamura K, Toyoda M, Hata K, Nakabayashi K, Umezawa A. Whole-exome sequencing of fibroblast and its iPS cell lines derived from a patient diagnosed with xeroderma pigmentosum. Genom Data. 2015;6:4-6.

33. Kalinderi K, Bostantjopoulou S, Fidani L. The genetic background of Parkinson's disease: current progress and future prospects. Acta Neurol Scand. 2016. doi:10.1111/ane.12563.

34. Li HF, Wu ZY. Genotype-phenotype correlations of amyotrophic lateral sclerosis. Trans Neurodegeneration. 2016:5:3.

35. Gatt JM, Burton KL, Williams LM, Schofield PR. Specific and common genes implicated across major mental disorders: a review of meta-analysis studies. J Psychiatr Res. 2015;60:1-13.

36. Li H, Durbin R. Fast and accurate short read alignment with BurrowsWheeler transform. Bioinformatics (Oxford, England). 2009;25(14):1754-60.

37. Li H, Handsaker B, Wysoker A, Fennell T, Ruan J, Homer N, Marth G, Abecasis G, Durbin R. The Sequence Alignment/Map format and SAMtools. Bioinformatics (Oxford, England). 2009;25(16):2078-9.

38. Isojima T, Doi K, Mitsui J, Oda Y, Tokuhiro E, Yasoda A, Yorifuji T, Horikawa R Yoshimura J, Ishiura $\mathrm{H}$, et al. A recurrent de novo FAM111A mutation causes Kenny-Caffey syndrome type 2. J Bone Miner Res Off J Am Soc Bone Miner Res. 2014;29(4):992-8.

39. Koh K, Ishiura H, Miwa M, Doi K, Yoshimura J, Mitsui J, Goto J, Morishita S, Tsuji S, Takiyama Y. Exome sequencing shows a novel de novo mutation in ATL1. Neuropsychiatry Clin Neurosci. 2014;2(1):1-4.

40. Okada Y, Matsumoto A, Shimazaki T, Enoki R, Koizumi A, Ishii S, Itoyama Y, Sobue G, Okano H. Spatiotemporal recapitulation of central nervous system development by murine embryonic stem cell-derived neural stem/ progenitor cells. Stem Cells (Dayton, Ohio). 2008;26(12):3086-98.

41. Kitada T, Asakawa S, Hattori N, Matsumine H, Yamamura Y, Minoshima S, Yokochi M, Mizuno Y, Shimizu N. Mutations in the parkin gene cause autosomal recessive juvenile parkinsonism. Nature. 1998;392(6676):605-8.

42. Tada H, Kuroki Y, Funabashi T, Kamiya Y, Goto T, Suyama K, Sano A, Mitsushima D, Etgen AM, Takahashi T. Phasic synaptic incorporation of GluR2-lacking AMPA receptors at gonadotropin-releasing hormone neurons is involved in the generation of the luteinizing hormone surge in female rats. Neuroscience. 2013;248:664-9.

43. Tada H, Okano HJ, Takagi H, Shibata S, Yao I, Matsumoto M, Saiga T, Nakayama Kl, Kashima H, Takahashi T, et al. Fbxo45, a novel ubiquitin ligase, regulates synaptic activity. J Biol Chem. 2010;285(6):3840-9.

44. Thorvaldsdottir H, Robinson JT, Mesirov JP. Integrative Genomics Viewer (IGV): high-performance genomics data visualization and exploration. Brief Bioinform. 2013;14(2):178-92.

\section{Submit your next manuscript to BioMed Central and we will help you at every step:}

- We accept pre-submission inquiries

- Our selector tool helps you to find the most relevant journal

- We provide round the clock customer support

- Convenient online submission

- Thorough peer review

- Inclusion in PubMed and all major indexing services

- Maximum visibility for your research

Submit your manuscript at www.biomedcentral.com/submit

) Biomed Central 